\title{
FRONTIÈRE D'UNE HYPERSURFACE PFAFFIENNE ET GÉOMÉTRIE SOUS-ANALYTIQUE
}

\author{
JEAN-MARIE LION et JEAN-PHILIPPE ROLIN \\ UMR5584 CNRS-Laboratoire de Topologie, Université de Bourgogne \\ BP400, 21011 Dijon cedex, France \\ E-mail:lion@u-bourgogne.fr; rolin@u-bourgogne.fr
}

Dédié au Professeur Stanistaw Lojasiewicz

Résumé. Nous donnons une nouvelle démonstration d'un théorème de Cano-Lion-Moussu : la frontière d'une hypersurface pfaffienne non spiralante est une réunion localement finie de sous-variétés analytiques connexes.

1. Introduction. Le cadre de notre étude est une variante de celui défini dans [M-R1,2]: on substitue aux objets semi-analytiques des objets sous-analytiques. Les théorèmes de $[M-R 1,2]$ restent vrais dans notre cadre qui est bien adapté aux projections linéaires $[\mathrm{Ro}]$ !

Soit $M$ un sous-analytique lisse relativement compact de $\mathbf{R}^{n}$ : c'est une sous-variété analytique de $\mathbf{R}^{n}$ qui est un sous-analytique relativement compact [Ło1-3]. On considère $\omega$ une 1-forme différentielle $\omega=a_{1} d x_{1}+\ldots+a_{n} d x_{n}$ dont les coefficients $a_{i}$ sont des fonctions analytiques au voisinage de $\bar{M}$. On suppose $\omega$ intégrable $(\omega \wedge d \omega \equiv 0)$, sans singularité dans $M\left(S(\omega) \cap M=\emptyset\right.$ où $\left.S(\omega)=\left\{a_{1}=\ldots=a_{n}=0\right\}\right)$ et transverse à $M\left(T_{x} M \not \subset \operatorname{ker} \omega(x)\right.$ si $\left.x \in M\right)$. La forme $\omega$ induit un feuilletage analytique $\mathcal{F}$ de codimension un de $M$. Suivant [M-R1,2], on dit qu'une feuille $V$ du feuilletage $\mathcal{F}$ est une feuille de Rolle si tout arc différentiable de $M$ et d'extrémités dans $V$ est tangent au feuilletage $\mathcal{F}$ en un point. Le triplet $(V, \omega, M)$ est appelé hypersurface pfaffienne de Rolle. Une feuille de Rolle $V$ de $M$ est une sous-variété analytique connexe et fermée de $M$. Elle est non-spiralante [M-R2]. Dès que $M$ est simplement connexe toute feuille de $\mathcal{F}$ est de Rolle [M-R2], [Hae]. Moussu et Roche [M-R1,2] (voir aussi [Kh]) prouvent que les feuilles de Rolle vérifient la propriété de finitude suivante.

1991 Mathematics Subject Classification: Primary 32B20; Secondary 34C35.

Received by the editors: November 4, 1996.

The paper is in final form and no version of it will be published elsewhere. 
ThÉorème De Finitude [M-R1,2]. Soient $\left(V_{1}, \omega_{1}, M_{1}\right), \ldots,\left(V_{p}, \omega_{p}, M_{p}\right)$ des hypersurfaces pfaffiennes de Rolle et soit $X \subset \mathbf{R}^{n}$ un sous-analytique relativement compact de $\mathbf{R}^{n}$. L'ensemble $V_{1} \cap \ldots \cap V_{p} \cap X$ a un nombre fini de composantes connexes.

Dans [L-Rl] on prouve, en adaptant les arguments de Wilkie [Wi] au cadre pfaffien "de Rolle", un théorème o-minimal pfaffien (suivant la terminologie de [vdD]).

THÉORÈME O-MINIMAL PFAFFIEN [L-Rl]. Il existe une classe de sous-ensembles relativement compacts des espaces $\mathbf{R}^{n}, n \in \mathbf{N}$, appelée classe des $\mathrm{T}^{\infty}$-pfaffiens, contenant les hypersurfaces pfaffiennes, stable par intersection, union, différence, projection linéaire, fermeture topologique. De plus, les composantes connexes d'un $\mathrm{T}^{\infty}$-pfaffien sont en nombre fini et sont des $\mathrm{T}^{\infty}$-pfaffiens.

A priori, la classe des $\mathrm{T}^{\infty}$-pfaffiens est plus large que celle des sous-pfaffiens qui est la plus petite classe de sous-ensembles des espaces $\mathbf{R}^{n}, n \in \mathbf{N}$ contenant les hypersurfaces pfaffiennes et stable par intersection, réunion, projection linéaire. On conjecture que les conclusions du théorème précédent sont vraies pour la classe des sous-pfaffiens. Dans [L-Rc] on prouve partiellement cette conjecture si $n \leq 3$. Le second résultat dans ce sens est le théorème suivant de [C-L-M].

ThÉORÈme 1 [C-L-M]. Si $(V, \omega, M)$ est une hypersurface pfaffienne de Rolle si $X$ est un sous-analytique alors $\bar{V} \cap X$ est une réunion localement finie de sous-variétés analytiques lisses connexes. C'est le cas de l'adhérence $\bar{V}$ et de la frontière $\bar{V} \backslash V$ de $V$.

L'objet de ce travail est de donner une nouvelle démonstration du théorème précédent. On utilise une version très faible du théorème de réduction pour les feuilletages analytiques de codimension un ([C-M] et appendice de $[\mathrm{C}-\mathrm{L}-\mathrm{M}])$. Le point nouveau est le lemme 2. Sa preuve s'inspire du lemme d'isotopie de Thom [Th]. Ce point de vue n'est pas loin de celui de Chazal [Chz1,2]. Nous utilisons le théorème d'uniformisation de Hironaka $[\mathrm{Hi1}, 2]$ (voir aussi $[\mathrm{B}-\mathrm{M}]$ ) et le théorème de stratification des applications de Hardt [Har] adapté au cadre pfaffien (voir [Ro]).

ThÉORÈme D'UniformisATion [Hi1,2]. Soit X un sous-analytique relativement compact de dimension $k$ de $\mathbf{R}^{n}$. Il existe $m \in \mathbf{N}$, une sous-variété différentiable analytique $U^{\prime}$ de dimension $k$ contenue dans $\mathbf{R}^{n+m}$, un sous-analytique lisse relativement compact de dimension $k, X^{\prime}$ inclus dans $\mathbf{R}^{n+m}$, tels que la fermeture $\overline{X^{\prime}}$ de $X^{\prime}$ soit contenue dans $U^{\prime}$ et l'image $\pi\left(X^{\prime}\right)$ de $X^{\prime}$ par la projection canonique de $\mathbf{R}^{n+m}$ sur $\mathbf{R}^{n}$ soit l'ensemble $X$.

ThÉORÈme DE HARdT PFAFFiEn [Har], [Ro]. Soient $Y_{1}, \ldots, Y_{r}$ des sous-analytiques relativement compacts de $\mathbf{R}^{n+1}$ et $\omega_{1}, \ldots, \omega_{p}$ des 1-formes différentielles à coefficients analytiques au voisinage de la fermeture $\bar{Y}$ de la réunion $Y=Y_{1} \cup \ldots \cup Y_{r}$. Soient $X_{1}, \ldots, X_{s}$ des sous-analytiques de $\mathbf{R}^{n}$ inclus dans l'image $X=\pi(Y)$ de $Y$ par la projection canonique $\pi$ de $\mathbf{R}^{n+1}$ sur $\mathbf{R}^{n}$. Il existe des stratifications $\mathcal{S}=\left\{\Gamma_{i}\right\}$ et $\mathcal{S}^{\prime}=\left\{\Gamma_{j}^{\prime}\right\}$ sous-analytique finies de $Y$ et $X$ vérifiant les propriétés suivantes. Les ensembles $Y_{k}$, $k=1, \ldots, r, S\left(\omega_{h}\right) \cap X, h=1, \ldots, p$ (resp. $\left.X_{l}, l=1, \ldots, s\right)$ sont des réunions de strates de $\mathcal{S}$ (resp. $\left.\mathcal{S}^{\prime}\right)$. Soit $\Gamma_{i}$ une strate de $\mathcal{S}$. Il existe une strate $\Gamma_{j}^{\prime}$ de $\mathcal{S}^{\prime}$ telle que $\pi\left(\Gamma_{i}\right)=\Gamma_{j}^{\prime}$ et la restriction $\pi: \Gamma_{i} \rightarrow \Gamma_{j}^{\prime}$ est un isomorphisme ou une fibration conjuguée à la projection canonique de $\left.\Gamma_{j}^{\prime} \times\right] 0,1\left[\right.$ sur $\Gamma_{j}^{\prime}$. Soit $h=1, \ldots, p$. Le noyau de la forme $\omega_{h}$ est transverse 
ou tangent à $\Gamma_{i}$ en tout point de $\Gamma_{i}$. De même, $T_{y} \Gamma_{i} \cap \operatorname{ker} \omega_{h}(y) \subset T_{y} \Gamma_{i} \cap \operatorname{ker} d \pi(y)$ en tout point de $\Gamma_{i}$, ou $T_{y} \Gamma_{i} \cap \operatorname{ker} \omega_{h}(y) \not \subset T_{y} \Gamma_{i} \cap \operatorname{ker} d \pi(y)$ en tout point de $\Gamma_{i}$.

Remerciements. Le premier auteur remercie S. Łojasiewicz, M. Kwieciński, W. Pawłucki et J. Stasica pour leur accueil toujours chaleureux à l'Université de Cracovie. Les deux auteurs remercient F. Cano, F. Chazal, O. Couture, R. Moussu, C. Roche, F. Ronga et L. van den Dries avec lesquels ils ont eu de nombreuses discussions qui leur ont permis de mettre au point ce travail.

2. Preuve du Théorème 1. Prouvons un énoncé plus précis que le Théorème 1.

ThÉorÈme 2. Soit $(V, \omega, M)$ une hypersurface pfaffienne de Rolle de $\mathbf{R}^{n}$ et $X$ un sousanalytique relativement compact. Il existe des sous-analytique lisses $X_{1}, \ldots, X_{s}$ inclus dans $\bar{X}$ dont la réunion contient $\bar{V} \cap X$ et vérifiant:

$(*)$ Soit $j=1, \ldots$, s. Il existe $r_{j} \in \mathbf{N}$ et pour $i=1, \ldots, r_{j}$ un sous-analytique $Y_{j}^{i} \subset$ $\mathbf{R}^{n+m_{j}^{i}}$, lisse et relativement compact et une hypersurface pfaffienne de Rolle $\left(V_{j}^{i}, \omega_{j}^{i}, Y_{j}^{i}\right)$ tels que la projection canonique $\pi_{j}^{i}$ de $Y_{j}^{i}$ sur $\mathbf{R}^{n}$ soit un isomorphisme analytique entre $Y_{j}^{i}$ et $X_{j}$ et $\bar{V} \cap X_{j}$ soit la réunion de $\pi_{j}^{1}\left(V_{j}^{1}\right) \cup \ldots \cup \pi_{j}^{r_{j}}\left(V_{j}^{r_{j}}\right)$ et d'un nombre fini de composantes connexes de $X_{j} \backslash\left(\pi_{j}^{1}\left(V_{j}^{1}\right) \cup \ldots \cup \pi_{j}^{r_{j}}\left(V_{j}^{r_{j}}\right)\right)$.

Remarques. On déduit du Théorème de Hardt pfaffien, du Théorème o-minimal pfaffien et du Théorème 2 que si $(V, \omega, M)$ est une hypersurface pfaffienne de Rolle de $\mathbf{R}^{n+m}$ alors l'image $\pi(V)$ de $V$ par la projection canonique de $\mathbf{R}^{n+m}$ sur $\mathbf{R}^{n}$ vérifie les conclusions du Théorème 2. La finitude dans les Théorèmes 1 et 2 est due au Théorème o-minimal pfaffien.

Soit $(V, \omega, M)$ une hypersurface pfaffienne de Rolle. Le problème est local. Quitte à diviser localement les 1-formes considérées par le pgcd de leurs cofficients on peut supposer que leurs lieux singuliers sont de dimension $n-2$.

Le lemme suivant est une synthèse de résultats de Cano, Lion, Moussu, Roche adaptés au cadre sous-analytique. La preuve est laissée au lecteur. Elle se déduit de l'existence de stratifications en feuillets normaux adaptés à des 1-formes différentielles analytiques [M-R1,2], du lemme de sélection de courbe pfaffien [Li1] et [L-Rc], du lemme de l'aile pfaffienne [Li2], [C-L-M] et de l'existence de triangulation sous-analytique [Ło1-3]. L'affirmation nouvelle est le point 4) qui est une variante du lemme de l'aile pfaffienne.

Lemme 1. Soit $(V, \omega, M)$ une hypersurface pfaffienne de Rolle, $X$ un sous-analytique inclus dans $\bar{M} \backslash M$ et $Y$ un sous-analytique de $\mathbf{R}^{n}$ inclus dans $M$. Il existe une stratification finie de $\bar{M}$ en sous-analytiques lisses qui vérifient les conditions suivantes.

1) Si $\Gamma$ est une strate

i) $\Gamma$ est adaptée aux ensembles $S(\omega), M, X, Y$ : chacun d'eux contient $\Gamma$ ou est disjoint de $\Gamma$.

ii) $\Gamma$ est transverse à $\omega$ en tout point ou tangente à $\omega$ en tout point: l'ensemble $\left\{x \in \Gamma / T_{x} \Gamma \subset \operatorname{ker} \omega(x)\right\}$ est vide ou égal à $\Gamma$.

2) $\bar{V} \cap Z=\overline{(V \cap A)} \cap Z$ si $A$ est la réunion des strates de dimension au plus $n-1$ contenues dans $M$ et $Z$ la réunion des strates de dimension au plus $n-3$ contenues dans $\bar{M} \backslash M$ (aile pfaffienne). 
3) Si $\Gamma$ est une strate de dimension $n-1$ alors $\Gamma$ est simplement connexe.

4) Supposons $M$ ouvert. Soient $\Gamma \subset \bar{M} \backslash M$ de dimension $n-2$ contenue dans le bord de $M$ et $\Gamma^{\prime} \subset M$ de dimension $n$ deux strates incidentes $: \Gamma \subset \overline{\Gamma^{\prime}}$. Il existe des applications sous-analytiques $f_{1}, \ldots, f_{n}, C^{\infty}$ sur un voisinage sous-analytique $U$ de $\Gamma^{\prime}$, telles que si on pose $f=\left(f_{1}, \ldots, f_{n}\right), g=\left(f_{3}, \ldots, f_{n}\right), h=f_{1}^{2}+f_{2}^{2}$ alors $g_{\mid \Gamma}$ est un plongement, $\Gamma \subset\left\{h=0, d f_{1} \wedge \ldots \wedge d f_{n} \neq 0\right\}$,

$$
\Gamma^{\prime} \subset\left\{h>0, d f_{1} \wedge \ldots \wedge d f_{n} \neq 0, \omega \wedge d h \wedge d f_{3} \wedge \ldots \wedge d f_{n} \neq 0\right\} .
$$

Si $\varepsilon \in \mathbf{R}^{n-2}, g^{-1}(\varepsilon) \cap \Gamma^{\prime}$ coupe $V$ transversalement: l'intersection $g^{-1}(\varepsilon) \cap \Gamma^{\prime} \cap V$ est une union finie de courbes analytiques connexes et fermées dans $\Gamma^{\prime}$ en restriction desquelles $f$ est strictement monotone.

Preuve du Théorème 2. On fait une récurrence sur la dimension de $M$. Les étapes 1 à 6 ramènent la preuve du Théorème 2 à un lemme. Ces étapes reprennent la réduction du théorème principal de $[\mathrm{C}-\mathrm{L}-\mathrm{M}]$ sans l'usage du Théorème de réduction pour les feuilletages analytiques de codimension un [C-M], [C-L-M].

Étape 1. Le résultat est connu si $M$ est de dimension 1 (Théorème de finitude uniforme).

Supposons avoir prouvé le Théorème 2 pour toute hypersurface pfaffienne $\left(V^{\prime}, \omega^{\prime}, M^{\prime}\right)$ où $M^{\prime}$ est de dimension inférieure strictement à celle de $M$ et prouvons le Théorème 2 pour $(V, \omega, M)$.

Étape 2. On déduit du Théorème d'uniformisation d'Hironaka, du Théorème de Hardt pfaffien, du Théorème o-minimal pfaffien et de l'hypothèse de récurrence qu'on peut supposer $M$ être un ouvert sous-analytique: $\operatorname{dim} M=\operatorname{dim} \mathbf{R}^{n}=n$.

Étape 3. D'après le théorème de finitude uniforme et l'affirmation 1) du lemme 1 , si $\Gamma \subset M$ est une strate alors $\Gamma \cap V$ est soit vide, soit confondue avec $\Gamma$, soit une réunion finie de feuilles de Rolle du feuilletage induit par $\omega$ sur $\Gamma$.

Étape 4. On déduit de l'hypothèse de récurrence appliquée à l'aile $A$ du lemme 1 (affirmation 2) que $Z \cap \bar{V}=Z \cap \overline{(A \cap Z)}$ vérifie les conclusions du théorème 2 si $Z$ est la réunion des strates de dimension au plus $n-3$ contenues dans $\bar{M} \backslash M$.

Étape 5. Soit $\Gamma$ une strate de dimension $n-1$ contenue dans $\bar{M} \backslash M$. On sait d'après le Théorème o-minimal pfaffien (voir aussi [L-Rc]) que $\bar{V} \cap \Gamma$ possède un nombre fini de composantes connexes. En raison des dimensions respectives de $\Gamma$ et $S(\omega)$, l'intersection $\Gamma \cap S(\omega)$ est vide. Si $\Gamma$ est tangente à $\omega$ alors $\bar{V} \cap \Gamma$ est vide. Supposons $\Gamma$ transverse à $\omega$. On déduit de l'affirmation 3) du lemme 1 que chaque composante connexe de $\bar{V} \cap \Gamma$ est une feuille de Rolle du feuilletage induit par $\omega$ sur $\Gamma$.

Étape 6 . Il reste à étudier $\bar{V} \cap \Gamma$ quand $\Gamma$ est une strate de dimension $n-2$ contenue dans $\bar{M} \backslash M$. On note $A_{1}$ et $A_{2}$ les réunions des strates de dimension $n-1$ de $M$ et $\bar{M} \backslash M$. D'après l'étape 3 et l'hypothèse de récurrence, $\Gamma \cap \overline{\left(A_{1} \cap V\right)}$ vérifie les conclusions du Théorème 2. D'après l'étape 5 et l'hypothèse de récurrence, $\Gamma \cap \overline{\left(A_{2} \cap \bar{V}\right)}$ vérifie les conclusions du Théorème 2 .

Le lemme suivant suffit pour conclure la preuve du Théorème 2 . Il remplace l'argument de réduction des feuilletages analytiques de codimension un de [C-L-M]. 
Lemme 2. L'ensemble $(\Gamma \cap \bar{V}) \backslash\left(\overline{\left(A_{1} \cap V\right)} \cup \overline{\left(A_{2} \cap \bar{V}\right)}\right)$ est la réunion de composantes connexes de $\Gamma \backslash\left(\overline{\left(A_{1} \cap V\right)} \cup \overline{\left(A_{2} \cap \bar{V}\right)}\right)$.

Preuve. Soit $\Gamma^{\prime}$ une strate ouverte dont l'adhérence contient $\Gamma$ et soit $a$ un point de $\left(\Gamma \cap \overline{\left(\Gamma^{\prime} \cap V\right)}\right) \backslash\left(\overline{\left(A_{1} \cap V\right)} \cup \overline{\left(A_{2} \cap \bar{V}\right)}\right)$. Pour prouver le lemme 2 il suffit de prouver que tout point $a^{\prime}$ de $\Gamma$ voisin de $a$ est dans $\left(\Gamma \cap \overline{\left(\Gamma^{\prime} \cap V\right)}\right) \backslash\left(\overline{\left(A_{1} \cap V\right)} \cup \overline{\left(A_{2} \cap \bar{V}\right)}\right)$.

On note $\delta_{a}$ la fonction définie par $\delta_{a}(x)=\left(f_{3}(x)-f_{3}(a)\right)^{2}+\ldots+\left(f_{n}(x)-f_{n}(a)\right)^{2}$. Il existe $\varepsilon>0$ tel que si $B_{a}(\varepsilon)$ désigne la composante connexe de $\left\{\delta_{a}(x)+h(x)<\varepsilon\right\}$ qui contient $a$ alors $B_{a}(\varepsilon) \cap\left(\overline{\left(A_{1} \cap V\right)} \cup \overline{\left(A_{2} \cap \bar{V}\right)}\right)$ est vide. Si $x$ est un point de $B_{a}(\varepsilon) \cap$ $\Gamma^{\prime} \cap V$ (il en existe puisque $a \in \overline{\left(\Gamma^{\prime} \cap V\right)}$ ) on note $C(x)$ la composante connexe de $B_{a}(\varepsilon) \cap \Gamma^{\prime} \cap\{g=g(x)\} \cap V$ qui contient le point $x$. D'après l'affirmation 4) du lemme 1, c'est une courbe analytique fermée de $B_{a}(\varepsilon) \cap \Gamma^{\prime}$ en restriction de laquelle la fonction $h=f_{1}^{2}+f_{2}^{2}$ est strictement monotone. On en déduit, en utilisant le lemme du petit chemin pfaffien [Li1], [L-Rc] que $\overline{C(x)} \backslash C(x)$ est formé de deux points, $a(x)$ et $b(x)$ qui vérifient $g(x)=g(a(x))=g(b(x)), a(x) \in \Gamma, b(x) \in \Gamma^{\prime} \cap V$ et $h(b(x))=\varepsilon-\delta_{a}(x)=\varepsilon-\delta_{a}(b(x))$. Il existe donc un point $c(x) \in C(x)$ tel que $h(c(x))=\left(\varepsilon-\delta_{a}(x)\right) / 2$. Par hypothèse il existe une suite $\left(x_{k}\right)_{k \in \mathbf{N}}$ de points de $\Gamma^{\prime} \cap V$ qui converge vers le point $a$. Quitte à considérer une sous-suite de $\left(x_{k}\right)_{k \in \mathbf{N}}$, on obtient une suite $\left(c\left(x_{k}\right)\right)_{k \in \mathbf{N}}$ associée qui converge vers un point $c$ de $\Gamma^{\prime} \cap V$ qui vérifie $g(c)=g(a)$. En reprenant le raisonnement précédent on montre que la composante connexe $C(c)$ de $B_{\varepsilon}(a) \cap \Gamma^{\prime} \cap\{g=g(c)\} \cap V$ qui passe par $c$ aboutit en $a: a \in \overline{C(c)} \backslash C(c)$. Puisque l'application $g$ est transverse à $\Gamma^{\prime} \cap V$ et que sa restriction $g_{\mid \Gamma}$ est un plongement (affirmation 4) du lemme 1), pour tout point $a^{\prime}$ de $\Gamma$ voisin de $a$ il existe un point $c^{\prime}$ de $B_{\varepsilon}(a) \cap \Gamma^{\prime} \cap V$ voisin de $c$ tel que $g\left(c^{\prime}\right)=g\left(a^{\prime}\right)$. Ainsi le point $a^{\prime}$ est dans l'adhérence de la composante connexe $C\left(c^{\prime}\right)$ de $B_{\varepsilon}(a) \cap \Gamma^{\prime} \cap\left\{g=g\left(c^{\prime}\right)\right\} \cap V$ qui contient $c^{\prime}$. Ceci achève la preuve du lemme 2 .

\section{Références}

[B-M] E. Bierstone et P. Milman, Semi-analytic and subanalytic sets, Inst. Hautes Études Sci. Publ. Math. 67 (1988), 5-42.

[C-L-M] F. Cano, J.-M. Lion et R. Moussu, Frontière d'une hypersurface pfaffienne, Ann. Sci. École Norm. Sup. (4) 28 (1995), 591-646.

[C-M] F. Cano et J.-F. Mattei, Hypersurfaces intégrales des feuilletages holomorphes, Ann. Inst. Fourier (Grenoble) 42 (1992), 49-72.

[Chz1] F. Chazal, Un théorème de fibration pour les feuilletages algébriques de codimension un de $\mathrm{R}^{m}$, C. R. Acad. Sci. Paris Sér. I Math. 321 (1995), 327-330.

[Chz2] F. Chazal, Sur les feuilletages algébriques de Rolle, Comment. Math. Helv. 72 (1997), 411-425.

[vdD] L. van den Dries, o-minimal structures, in: Logic: from Foundations to Applications (Conference Proceedings), W. Hodges et al. (eds.), Oxford University Press, New York, 1996, 137-185.

[Hae] A. Haefliger, Structures feuilletées et cohomologie à valeurs dans un faisceau de groupoïdes, Thèse, Comment. Math. Helv. 32 (1958), 248-329. 
[Har] R. M. Hardt, Topological properties of subanalytic sets, Trans. Amer. Math. Soc. 211 (1975), 150-208.

[Hi1] H. Hironaka, Introduction to Real-analytic Sets and Real-analytic Maps, Istituto Matematico L. Tonelli, Pisa, 1973.

[Hi2] H. Hironaka, Subanalytic sets, in: Number Theory, Algebraic Geometry and Commutative Algebra in Honor of Yasuo Akizuki, Kinokuniya, Tokyo, 1973, 453-493.

[Kh] A. G. Khovanskii, Real analytic varieties with the finiteness property and complex abelian integrals, Functional Anal. Appl. 18 (1984), 119-127.

[Li1] J.-M. Lion, Partitions normales de Eojasiewicz et hypersurfaces pfaffiennes, C. R. Acad. Sci. Paris Sér. I Math. 311 (1990), 453-456.

[Li2] J.-M. Lion, Un lemme d'aile pour les ensembles pfaffiens, C. R. Acad. Sci. Paris Sér. I Math. 316 (1993), 187-189.

[L-Rc] J.-M. Lion et C. A. Roche, Topologie des hypersurfaces pfaffiennes, Bull. Soc. Math. France 124 (1996), 35-59.

[L-Rl] J.-M. Lion et J.-P. Rolin, Feuilletages analytiques réels et Théorème de Wilkie, préprint de l'Université de Bourgogne, 1996.

[Ło1] S. Łojasiewicz, Ensembles semi-analytiques, Inst. de Hautes Études Scientifiques, Bures-sur-Yvette, 1965.

[Ło2] S. Łojasiewicz, Triangulation of semi-analytic sets, Ann. Scuola Norm. Sup. Pisa (3) 18 (1964), 449-474.

[Ło3] S. Łojasiewicz, Stratifications et triangulations sous-analytiques, Universitá degli Studi di Bologna, 1986.

[M-R1] R. Moussu et C. A. Roche, Théorie de Hovanskii et problème de Dulac, Invent. Math. 105 (1991), 431-441.

[M-R2] R. Moussu et C. A. Roche, Théorèmes de finitude uniforme pour les variétés pfaffiennes de Rolle, Ann. Inst. Fourier (Grenoble) 42 (1992), 393-420.

[Ro] C. A. Roche, Densities for certain leaves of real analytic foliations, Astérisque 222 (1994), 373-387.

[Th] R. Thom, Ensembles et morphismes stratifiés, Bull. Amer. Math. Soc. (N.S.) 75 (1969), 240-284.

[Wi] A. Wilkie, Tangents to analytic varieties. A general theorem of the complement and some new o-minimal structures, preprint, 1996. 